Zum Schlusse ist es mir noch eine angenehme Pflicht, meinem verehrten Lehrer, Herrn Prof. Dr. J. Moeller, Vorstand des Pharmakologischen Instituts der Universität Graz, welcher die Güte hatte, sowohl Zeichnungen als Text einer Nachprüfung zu unterziehen, meinen Dank auszusprechen. Herr M. Silvermann führte die chemischen Analysen aus und Dr. C. E. Preston half mir beim mikroskopischen Präparieren. Fig. 30 wurde nach einer Photographie von W. E. Britten, Fig. 26 nach einer Photographie von J. F. Ma lone und die übrigen Schnitte wurden nach meinen Zeichnungen hergestellt.

\title{
Über das natürliche Vorkommen von Salicylsäure in Erdbeeren und Himbeeren.
}

Von

\section{Dr. Karl Windiseh.}

Mitteilung aus der önochemischen Versuchsstation zu Geisenheim a. Rh.

R. Hefelmann ${ }^{1}$ ) fand gelegentlich der Prüfung von Fruchtsäften auf Salicylsäure, daß es Himbeersäfte gibt, die bei der Untersuchung nach dem üblichen Verfahren Salicylsäure-Reaktionen geben, ohne daß ihnen dieses Konservierungsmittel zugesetzt worden ist. Er stellte fest, daß die "salicylsäureähnliche Substanz" destillierbar ist und dab sie aus den Kernen der Himbeeren herrühre. Die Isolierung dieser Substanz gelang Hefelmann nicht, auch wurde nicht der Nachweis erbracht, ob sie wirklich Salicylsäure sei oder nur dieser Säure ähnliche Reaktionen gebe.

Im Jahre 1901 machten Truchon und Martin-Claude ${ }^{2}$ ) ähnliche Beobachtungen bezüglich der Erdbeere und der daraus hergestellten Erzeugnisse. Sie untersuchten eine Anzahl Obstfrüchte (Kirschen, Erdbeeren, Himbeeren, rote, weiße und schwarze Johannisbeeren, Äpfel, Birnen, Quitten, Pfirsiche, Aprikosen, Reineclauden und Mirabellen) auf Salicylsäure, indem sie die Säfte mit einer Mischung von Äther und Petroleumäther ausschüttelten und den beim Verdunsten der Äthermischung hinterbleibenden Rückstand mit verdünnter Eisenchloridlösung prüften. Kirschen, Pfirsiche und Quitten gaben dabei grüne, die schwarzen Johannisbeeren rotbraune, zwei Sorten Erdbeeren aber deutliche violette Farbenreaktionen. Sie glaubten aber nicht, daß diese Violettfärbung durch Salicylsäure verursacht sei, sondern durch eine besondere Art von Gerbstoff. der den Erdbeeren eigentümlich sei und mit Eisenchlorid die violette, Salicylsäure vortäuschende Reaktion gebe.

Truchon und Martin-Claude hielten es deshalb für notwendig, bei der Prüfung von Erdbeeren und Erdbeer-Erzeugnissen auf Salicylsäure diesen Gerbstoff vorher zu entfernen, da er den Salicylsäurenachweis unsicher mache. Sie empfablen für diesen Zweck ein Verfahren, das von J. de Brévans ${ }^{3}$ ) für den Nachweis von Sac-

1) R. HefeImann, Zur Beurteilung der Fruchtsäfte und Fruchtsyrupe des Handels und über den Nachweis der Salicylsäure in denselben. - Zeitsehr. öffentl. Chemie 1897, 3, 171.

7) Truchon et Martin-Claude, Sur la composition de certains jus de fruits destinés a la fabrication des confitures, sirops ete. - Annal. chim. analyt. 1901, 6, 85; diese Zeitschr. $1901,4,703$.

3) Annal. chim. analyt. 1900, 5, 131; diese Zeitschr. 1900, 4, 180. 
charin angewandt worden war. Nach diesem Verfahren werden $100 \mathrm{ccm}$ Fruchtsaft mit $2 \mathrm{ccm}$ konzentrierter Eisenchloridlösung versetzt; man schüttelt die Mischung und trägt unter fortwährendem Schütteln $10 \mathrm{~g}$ Calciumkarbonat ein. Nach dem Absitzen filtriert man, schüttelt das meist farblose Filtrat mit Äther aus und prüft den Verdunstungsrückstand der Ätherlösung mit verdünnter Eisenchloridlösung. Durch diese Behandlung sollen die Gerbstoffe entfernt werden, welche die Salicylsäure-Reaktion stören können. Als Truchon und Martin-Claude Erdbeersaft nach diesem Verfahren behandelten, erhielten sie tatsächlich keine Spur einer Salicylsäure-Reaktion; als sie aber den Erdbeersaft mit 0,1 g Salicylsäure auf 1 Liter Wasser versetzten, erhielten sie unter den gleichen Bedingungen eine starke Salicylsäure-Reaktion. Sie glaubten damit bewiesen zu haben, daß die violette Eisenchlorid-Reaktion der Erdbeeren nicht dureh Salicylsäure, sondern durch einen Gerbstoff hervorgerufen werde.

Kurze Zeit später traten L. Portes und A. Desmoulières ${ }^{1}$ ) mit umfassenderen Untersuchungen über diesen Gegenstand hervor. Sie prüften 10 Sorten Erdbeeren, die sie teils selbst gezogen, teils angekauft hatten, in versehiedener Weise auf Salicylsäure. Sie zogen die zerriebenen Erdbeeren mit Wasser aus, kochten sie mit Zuckersirup oder mit Kalilauge; in allen Fällen erhielten sie nach dem Ausschütteln mit Äther-Petroleumäther deutliche und starke Salicylsäure-Reaktionen. Mehrere andere Salicylsäure-Reaktionen (Rotfärbung mit Salpetersäure, A mmoniak und Eisenchlorid, Gelbfärbung mit Schwefelsäure, Salpetersäure und Ammoniak, Rotfärbung mit Essigsäure, Kaliumnitrit und Kupfersulfat nach Jori ssen, schwacher Niederschlag mit Bromwasser) traten ebenfalls deutlich ein. Um den Beweis zu erbringen, daß die violette Reaktion nicht durch einen Gerbstoff verursacht wird, fällten Portes und Desmoulières aus einer größeren Menge Erdbeersaft die Gerbstoffe mit einer ammoniakalischen Lösung von Zinkacetat aus. Im Filtrat wurde eine deutliche Salicylsäure-Reaktion erhalten, während die durch Schwefelwasserstoff aus der Zinkverbindung frei gemachten Gerbstoffe der Erdbeere mit Eisenchlorid eine braunschwarze Färbung ergaben.

Diese Beobachtung stebt im Widerspruche mit der Annahme von Truchon und Martin-Claude, daß die violette Reaktion der Erdbeere durch einen Gerbstoff bewirkt werde. Portes und Desmoulières konnten die Unrichtigkeit dieser Annahme beweisen. Sie stellten fest, daß beim Behandeln einer Salicylsäure enthaltenden Flüssigkeit mit Eisenchlorid und Caleiumkarbonat die Salicylsäure in ein Salz (wobl Caleiumsalz) übergeführt wird und als solches in das Filtrat geht. Wenn man letzteres ohne weiteres mit Äther ausschüttelt, so geht natürlich keine Salicylsäure in den Äther, weil nur die freie Salicylsäure, nicht aber ihre Salze in Äther lößlich sincl. Sobald man jedoch das Filtrat mit Schwefelsäure ansäuert und dann mit Äther aussehüttelt, geht die Salicylsäure in den Äther über und gibt eine starke Reaktion. Als Portes und Desmoulières den Erdbeersaft in dieser Weise behandelten, erhielten sie wiederum eine deutliche Salicylsäure-Reaktion. Aus einer größeren Menge Erdbeersaft konnten sie nach dem Kochen mit Kali Krystalle abscheiden, die unter dem Mikroskop den Salicylsäure-Krystallen durchaus glichen. Die kolorimetrische Bestimmung ergab in $1 \mathrm{~kg}$ Erdbeeren etwa $1 \mathrm{mg}$ Salicylsäure.

Durch diese Untersuchungen schien die Gegenwart von Salicylsäure in den Erd-

1) L. Portes et A. Desmoulières, Présence normale de l'acide salicylique dans les fraises; erreurs d'analyses qui peuvent en résulter. - Annal. chim. analyt. 1901, 6, 401; diese Zeitschr. 1902, 5, 468. 
beeren sicher nachgewiesen zu sein. Dem widerspricht jedoch eine kürzlich erschienene Mitteilung von G. Paris's), der drei Sorten italienischer Erdbeeren aus der Provinz Avellino untersuchte und ausdrücklich erklärte, sie seien frei von Salicylsäure.

Im Sommer 1902 habe ich Gelegenheit genommen, eine Anzahl Obstfrichte auf das Vorkommen von Salicylsäure zu prüfen. Die Untersuchung erstreckte sich auf mehrere Sorten Kirschen, mehrere Sorten Pflaumen, Reineclauden, Mirabellen, Aprikosen, Pfirsiche, rote, weibe und schwarze Johannisbeeren, rote und weiße Stachelbeeren, Himbeeren und Erdbeeren und die bisweilen zur Herstellung von Fruchtsäften und Likörweinen benntzten Mahoniabeeren. Die Vorversuche wurden in folgender Weise ausgeführt: Größere Mengen der Früchte wurden auf der Obstmühle gemahlen und auf einer kleinen Kelter abgepreßt. Die Fruchtsäfte wurden mit etwa $2 \%$ konzentrierter Schwefelsäure versetzt und am Rückflußkühler ${ }^{1 / 4}$ Stunde gekocht; dadurch sollten etwa vorhandene Ester der Salicylsäure verseift werden. Nach dem Erkalten wurde der Kühler mit Äther ausgespült und die Fruchtsäfte zweimal mit größeren Mengen Äther ausgeschüttelt. Man hob die Ätherschichten ab, schüttelte sie mit etwas Natronlauge tüchtig dureh und destillierte den Ather auf einem elektrisch geheizten Wasserbade ab. Aus dem Destillationsrückstande wurde der letzte Rest des Äthers auf dem Wasserbade abgedampft, der Rückstand mit Wasser in einen kleinen Scheidetrichter gespült, mit Schwefelsäure angesäuert und zweimal mit Benzol ausgeschüttelt. War die Benzollösung farblos, so wurde das Benzol bei gewöhnlicher Temperatur verdunstet und der Rückstand mit stark verdünnter Eisenchloridlösung auf Salicylsäure geprüft. War die Benzollösung gelb gefärbt, so wurde sie nochmals mit Natronlauge durchgeschüttelt, das Benzol abdestilliert, der Rüclsstand mit Wasser aufgenommen und nach dem Ansäuern mit Schwefelsäure nochmals mit Benzol ausgeschüttelt. Die Benzollösung wurde dann bei gewöhnlicher Temperatur verdunstet und der Rückstand auf Salicylsäure geprüft.

Von den vorher genanten Obstarten gaben nur Himbeeren und Erdbeeren eine deutliche violette Salicylsäure-Reaktion; bei den übrigen Obst- und Beerenarten wurde nicht eine Andeutung einer Reaktion erhalten. Auf Grund dieser Vorversuche wurden nun weitere Untersuchungen mit Himbeeren und Exdbeeren ausgeführt. Zunächst wurden die Fruchtsäfte einmal direkt mit $\overparen{\text { ther }}$ und Benzol ausgeschüttelt und das andere MaI nach dem Kochen mit Schwefelsäure bezw. Natronlauge. Stets wurde die Salicylsäure-Reaktion bei den verseiften Fruchtsäften erbeblich stärker erbalten als bei den direkt behandelten Säften. Damit ist bewiesen, daß wenigstens ein großer Teil der Salicylsäure in gebundener Form, vielleicht als Ester, in den Früchten enthalten ist.

Zur weiteren Kennzeichnung der Salieylsäure wurde der Benzol-Rückstand nach dem Verfahren von A. Jorissen mit Essigsäure, Kaliumnitrit und Kupfersulfat geprüift; es trat bei Erdbeeren und Himbeeren die rote Salicylsäure-Reaktion ein. In einer anderen Versuchsreihe wurden die Benzol-Rückstände mit verdünnter Natronlauge aufgenommen, in einem Destillierkölbchen mit Schwefelsäure wieder angesäuert und destilliert. Das Destillat wurde mit Äther ausgeschültelt, der Äther bei gewöhnlicher Temperatur verdunstet und der Rückstand mit verdünnter Eisenchloridlösung auf Salicylsäure geprüft; es trat eine deutliche starke Violettfärbung auf. Nach Maßogabe dieser

1) G. Paris, Chemische Zusammensetzung der Fragaria vesca. - Chem.-Ztg. 1902, 26, 248; diese Zeitschr. 1908, 6, 29. 
Ergebnisse ist kein Zweifel mehr möglich, daß die Himbeeren und Erdbeeren kleine Mengen Salicylsäure enthalten; namentlich die Flüchtigkeit der die violette Eisenchlorid-Reaktion gebenden Substanz mit Wasserdämpfen ist für die Salicylsäure charakteristisch.

Die Untersuchungen erstreckten sich nicht nur auf eine Varietät Erdbeeren und Himbeeren, sondern auf mehrere. Von jeder Beerenart wurden drei Proben verschiodener Herkunft geprüft. Eine Sorte Himbeeren stammte aus dem Garten der hiesigen Königlichen Lehranstalt für Wein-, Obst- und Gartenbau, die zweite (ebenfalls eine Gartenhimbeere) wurde von auswärts bezogen, die dritte war eine wild gewachsene Waldhimbeere. Die Erdbeeren rührten teilweise aus dem Garten der genannten Lehranstalt, teilweise von dem Versuchsfelde der önochemischen Versuchsstation her; die dritte Sorte war eine Walderdbeere. Inwieweit die Gartenerdbeeren und Gartenhimbeeren bezüglich der Varietät einheitlich waren, vermag ich nicht zu beurteilen; vermutlich bestanden sie aus Mischungen verschiedener Varietäten. Die Untersuchungen ergaben, daß alle Proben Salicylsäure enthielten, die im Garten gezüchteten jedoch erheblich mehr als die wild gewachsenen Waldhimbeeren und Walderdbeeren.

In bezug auf die Menge der Salicylsäure in den beiden Beerenobstarten ergab sich in allen Fällen, daß die Erdbeeren weit reicher an dieser Substanz waren als die Himbeeren. Um eimen annähernden Maßstab für ibren Salicylsäuregebalt zu ge. winnen, wurde eine kolorimetrische Bestimmung der Salicylsäure vorgenommen. Je zwei Liter Erdbeersaft und Himbeersaft (Gartenfrüchte) wurden mit 2\% Schwefelsäure am Rückflußkühler 1/4 Stunde gekocht, nach dem Erkalten zweimal mit Äther ausgeschüttelt und weiter verfahren, wie bei der qualitativen Prüfung der Fruchtsäfte. Die Benzol-Rückstände wurden in warmem Wasser gelöst und die Stärke der Eisenchlorid-Reaktion kolorimetrisch mit der verglichen, die mit sehr dünnen SalicylsäureLösungen von bekanntem Gehalte erhalten wurden. Es konnte hierbei festgestellt werden, daß der Farbenton der Eisenchlorid-Reaktion der Fruchtsaft-Auszüge vollständig mit dem Farbentone der Reaktion mit reiner Salicylsäure übereinstimmte. Nach Maßgabe dieser kolorimetrischen Bestimmungen enthielt der Himbeersaft 1,1 mg, der Erdbeersaft $2,8 \mathrm{mg}$ Salicylsäure im Liter. Da bei dem Ausschütteln der Salicylsäure kleine Verluste nicht zu vermeiden sind, ist der Salicylsäure-Gehalt der Exdbeeren und Himbeeren voraussichtlich noch etwas größer. Derselbe war bei den Erdbeeren so groß, daß die Salicylsäure in $50 \mathrm{ccm}$ mit Schwefelsäure gekochtem Erdbeerensaft nach dem beim Weine üblichen Verfahren scharf nachweisbar war; selbst beim Ausschütteln von $25 \mathrm{ccm}$ Erdbeersaft mit einer Mischung gleicher Raumteile Äther und Petroleumäther wurde noch eine schwache, aber deutliche violette Reaktion mit Eisenchlorid erhalten.

Von der Herstellung von Erdbeerwein und -saft stand eine größere Menge nur unvollständig ausgepreßter Erdbeertrester zur Verfügung. Diese wurden dazu benutzt, die Salicylsäure in Substanz aus den Erdbeeren zu isolieren. Die Trester wurden mit $2 \%$-iger Schwefelsäure übergossen, längere Zeit stehen gelassen und dann abgeprelat. Die Flüssigkeit wurde etwa literweise am Rückfluokühler gekocht und ihr in der vorher beschriebenen Weise mit Äther und Benzol die Salicylsäure entzogen. Die Benzollösungen wurden bei gewöbnlicher Temperatux stark eingedunstet, miteinander vereinigt, mit ein wenig Tierkohle geschüttelt und filtriert. Beim Verdunsten des Benzols in einem Schälchen hinterblieben neben einem stark riechenden ÖI dünne, weiße, stark glänzende Krystallnadeln, die, auch unter dem Mikroskop, ron einem eigens für diesen 
Zweck hergestellten Salicylsäure-Präparat nicht zu unterscheiden waren. Wie bei diesem saßen die Krystallnadeln aus den Erdbeeren nicht am Boden des Schälchens, sondern mehr nach dem Rande hin. Mit Eisenchlorid gaben sie eine rein violette Reaktion. Ein Teil der Nadeln wurde in Äther gelöst und der Äther in einem Probierröhrchen bei gewöhnlicher Temperatur verdunstet. Bei vorsïchtigem Erwärmen ließ sich der Verdunstungsrückstand in den oberen Teil des Probierröhrchens sublimieren; das Sublimat gab ebenfalls eine rein violette Eisenchlorid-Reaktion. Ein Teil der Krystallnadeln wurde in warmem Wasser gelöst und die Lösung mit einigen Tropfen stark verdünnten Bromwassers versetzt; es trat eine deutliche weiße Trübung auf. Auch diese Reaktion weist auf Salicylsäure hin, die mit Bromwasser einen weißen, unlöslichen Körper von der Formel $\mathrm{C}_{6} \mathrm{H}_{2} \mathrm{Br}_{4} \mathrm{O}$ gibt ${ }^{1}$ ):

$$
\mathrm{C}_{6} \mathrm{H}_{4}<\mathrm{COOH}+8 \mathrm{Br}=\mathrm{C}_{6} \mathrm{H}_{2} \mathrm{Br}_{3}-\mathrm{O}^{-} \mathrm{Br}+4 \mathrm{HBr}+\mathrm{CO}_{2} \text {. }
$$

Wenn auch die Menge der aus den Erdbeeren gewonnenen Substanz nicht hinreichte, um eine Schmelzpunktbestimmung oder eine Elementaranalyse auszuführen, so darf man doch mit Sicherheit annehmen, daß dieselbe wirklich Salicylsäure war.

Von Himbeeren standen ebenfalls größere Mengen mäßig ausgepreßter Trester zur Verfügung, die bei der Himbeersaftbereitung zurückgeblieben waren. Auch hier gelang es, Andeutungen von Krystallen mit den Eigenschaften der Salicylsäure zu isolieren, wenn auch in geringerer Menge und weniger charakteristisch als bei den Erdbeeren.

Was die Form, in der die Salicylsäure in den Erdbeeren und Himbeeren enthalten ist, betrifft, so scheint wenigstens ein Teil derselben in gebundenem, verseifbarem Zustande, also wohl in Esterform, vorhanden zu sein. Dafür spricht der Umstand, daß nach dem Kochen der Säfte mit Schwefelsäure oder Natronlauge stets eine stärkere Salicylsäure-Reaktion erhalten wurde als mit den natürlichen Säften. Auch Portes und Desmoulières $s^{2}$ ) neigen dieser Ansicht zu. Sie fanden, daß die violetten Eisenchlorid-Reaktionen, die mit den Auszügen aus den natürlichen Säften erhalten wurden, auf Zusatz von Chloroform verschwanden, während die EisenchloridReaktionen, die mit den Auszügen aus den verseiften Säften erhalten wurden, auf Zusatz von Chloroform bestehen blieben. Die erstere Erscheinung soll für Methylsalicylat bezw. für die Salicylsäureester charakteristich sein.

Die vorstehenden Untersuchungen lassen keinen Zweifel mehr bestehen, daß die Frdbeeren und Himbeeren und die aus diesen Beerenfrüchten hergestellten Erzeugnisse (Wein, Syrup, Gelee, Marmelade u. s. w.) einen geringen natürlichen Gehalt an Salicylsäure enthalten. Es wird nummehr festzustellen sein, ob die Salicylsäure als ein normaler Bestandteil dieser Beerenfrüchte anzusehen ist, $d$. b. ob sie regelmäßig in ihnen auftritt. Zu dem Zwecke wäre es wünschenswert, daß die Prüfung der Erdbeeren und Himbeeren auf Salicylsäure an recht vielen Stellen vorgenommen würde. Bestätigt sich der Befund, so wird die Nahrungsmittel-Kontrolle ihre Folgerungen daraus ziehen müssen. Es wird dann nicht mehr angängig sein, ein Erdbeeroder Himbeererzeugnis wegen eines sehr geringen Salicylsäure-Gehaltes ohne weiteres zu beanstanden. Man wird vielmehr versuchen müssen, festzustellen, ob die Salicylsäure dem Erzeugnis zugesetzt worden ist. Hierbei wird die quantitative Bestim-

1) R. Benedikt, Liebig's Annalen der Chemie 1879, 199, 135.

2) Annal. chim. analyt. 1901, 6, 401; diese Zeitschr. 1902, 5, 468. 
mung der Salicylsäure eine Rolle spielen, da der natürliche Gehalt der Erdbeer- und Himbeer-Erzeugnisse an Salicylsäure sehr gering ist, während ein zur Konservierung gemachter Salicylsäure-Zusatz, wenn er seinen Zweck erfüllen und wirklich konservierend wirken soll, sehr viel größer sein muß. Schon jetzt ist bei der Beurteilung. kleiner Salicylsäuremengen in diesen Erzeugnissen Vorsicht anzuraten.

Nachschrift. Nach Abschluf der vorstehenden Untersuchungen ersah ich aus Heft 40 der „Zeitschr. für angewandte Chemie“ (1902, 15, 1041), daß P. S ü ß auf der Versammlung deutscher Naturforscher und Ärzte in Karlsbad am 25. Sept. 1902 einen Vortrag über dasselbe Thema gehaiten hat. $\mathrm{S} \ddot{3} ß$ stellte ebenfalls in Garten- und Walderdbeeren Salicylsäure in Mengen von 2 bis $3 \mathrm{mg}$ in 1 Liter Saft fest; er erhielt Krystallnadeln, die sublimierbar und destillierbar waren und mit Eisenchlorid eine violette Reaktion gaben. In Johannisbeeren, Stachelbeeren, Brombeeren, Kirschen, Pflaumen, Reineclauden, Äpfeln und Birnen konnte Salicylsäure nicht nachgewiesen werden. Die Wahrscheinlichkeit, daß die Salicylsäure ein normaler Bestandteil der Erdbeeren ist, wird durch diese Ergebuisse wesentlich erhöht ${ }^{\mathbf{1}}$ ).

1) Einem ausführlichen Autoreferat von S ï $B$ in dieser Zeitschrift $(1902,5,1201-1204)$ ist zu entnehmen, daß3 er in Himbeeren Salicylsäure nicht finden konnte. Bei dieser Beerenart sind daher weitere Untersuchungen in den nächsten Jahren erforderlich.

\title{
Untersuchnng zweier neuerdings in den Handel gebrachten Klärmittel für Wein und Branntwein.
}

\author{
Von
}

\section{Dr. Karl Windisch.}

Mitteilung aus der önochemischen Versuchsstation zu Geisenheim a. Rh.

Die Klärung der Weine bietet in der Praxis nicht selten recht erhebliche Schwierigkeiten. Neben den bekannten und in der Kellerwirtschaft anerkannten Klärungsmitteln, den sogenannten Schönungsmitteln, tauchen von Zeit zu Zeit neue Mittel auf, denen, mit Recht oder Unrecht, eine besonders gute und sichere Wirkung zugeschrieben wird. Da die Abnehmer den größten Wert darauf legen, daß die Weine vollständig klar (blank) sind, so ist es leicht verständlich, daß die Weinhändler mit Vorliebe alles probieren, was ihnen von neuen Klärungsmitteln angeboten wird. Vielfach sind dies Geheimmittel, deren Zusammensetzung von den Fabrikanten nicht bekannt gegeben wird; es wird dann blo $\mathrm{B}$ in allgemeinen Redewendungen versichert, daß das Mittel nicht gegen das Nahrungsmittelgesetz und das Weingesetz verstoße und nicht beanstandet werden könne. Während manche Weinhändler solche Geheimmittel ohne Bedenken verwenden, sind andere vorsichtiger und lassen dieselben untersuchen, bevor sie sie gebrauchen. Auf diese Weise kam auch die önochemische Versuchsstation zu Geisenheim in den Besitz zweier Klärungsmittel für Wein und Spirituosen, die ihr innerhalb kurzer Zeit von verschiedenen Weinfirmen zur Untersuchung eingesandt wurden. 